\title{
Robotic Handwriting: Multi-contact Manipulation based on Reactional Internal Contact Hypothesis
}

\author{
Sung-Kyun $\mathrm{Kim}^{1,2}$, Joonhee $\mathrm{Jo}^{2}$, Yonghwan $\mathrm{Oh}^{2}$, Sang-Rok $\mathrm{Oh}^{2}$, Siddhartha Srinivasa ${ }^{1}$, Maxim Likhachev ${ }^{1}$ \\ ${ }^{1}$ The Robotics Institute, Carnegie Mellon University, USA \\ ${ }^{2}$ Interaction \& Robotics Research Center, Korea Institute of Science and Technology, Korea \\ kimsk@cs.cmu.edu, \{jhjo, oyh, sroh\}@kist.re.kr, \{siddh, maxim\}@cs.cmu.edu
}

\begin{abstract}
When one uses a hand-held tool, the fingers often make the tool to be in contact with the palm in the form of multi-contact manipulation. Multi-contact manipulation is useful for object-environment interaction tasks because it can provide both powerful grasping of the object body and dexterous manipulation of the object end-effector. However, dealing with the internal link contact with the object is not trivial. In this paper, we propose Reactional Internal Contact Hypothesis that regards the internal contact force as a reaction force so that the desired finger force can be reduced. By taking a handwriting task as an example, optimal configuration search and grasping force computation problems are addressed based on this hypothesis and validated via dynamic simulation.
\end{abstract}

\section{INTRODUCTION}

In our daily life, we use a variety of types of grasps, from simple pinching to complex grasp beyond the Cutkosky's taxonomy [1]. Robots are also extending their grasping ability in various forms to follow up humans.

Simple hands with limited number of fingers and degreeof-freedom (DOF) have had great success in grasping a variety of objects [2], [3], [4]. Under-actuated hands are now popular for their intrinsic compatibility during interaction with the object [5], [6], [7]. Works on fully-actuated, high degrees-of-freedom hands are still ongoing as well because of their unique dexterity and diversity [8], [9], [10].

Multi-contact manipulation which includes complex grasps and in-hand manipulation is one example that necessitates a multi-fingered hand. This appears frequently in our daily life when we use some tools, for example, handwriting, screw-driving, and chopstick handling. The interesting characteristics of multi-contact manipulation is that it provides both of powerful grasping of object body and dexterous manipulation of object end-effector, i.e., tooltip, simultaneously.

However, it is not easy to apply this multi-contact manipulation skill to a robot. The main challenge is in dealing with the internal link contact with the object. For fingertips, there are many options for sensors and actuators, yet internal links like the palm lack in ability to generate force actively or understand the interaction with the object accurately [11], [12].

There can be two approaches to cope with the internal link contact. One is to embed a high-resolution skin sensor all around the hand [13], [14]. The other is to estimate or

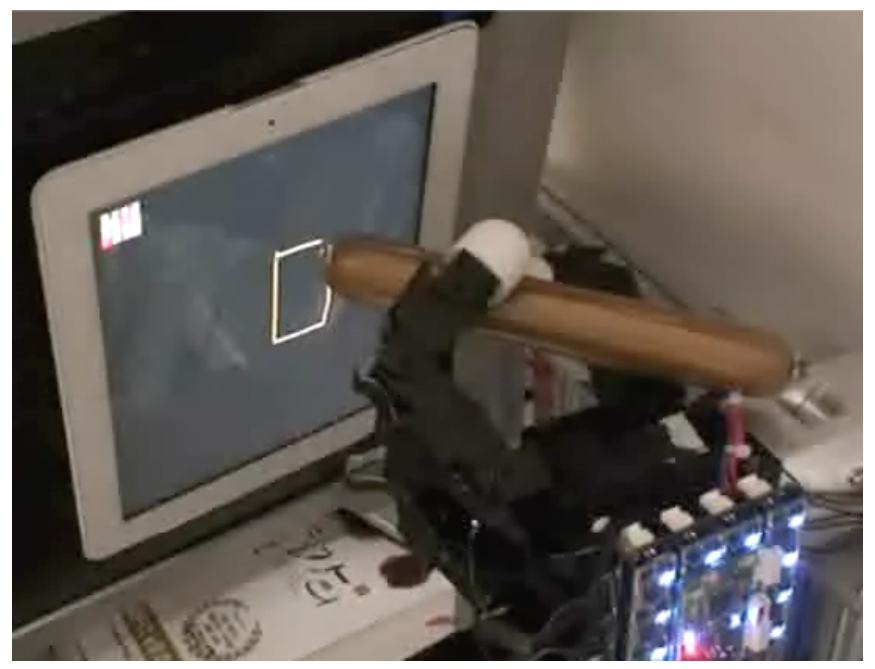

Fig. 1. An example of multi-contact manipulation for robotic handwriting (preliminary experiment).

compensate the internal contact effect using other attainable contact point information [15], [16]. This paper is closer to the second approach but proposes a novel method to take advantage of the internal contact rather than just to estimate it.

The key idea in this paper is to regard the force from the internal link contact as a reaction force that can make the system resistant to the external forces. A simple example of how reaction force gives resistance is depicted in Fig. 2. When the finger applies a force to the object that is in contact with immobile environment, the reaction force cancels the finger force out, and these two forces work as internal forces, that is, they make a sturdy grasp of the object but make no motion of it.

Notice that, unlike two constant active forces, one constant active force and one reaction force can make equilibrium in spite of external disturbance unless its magnitude is greater than that of the active force. This demonstrates the benefit of reaction force in grasping. This phenomenon that appears in multi-contact manipulation will be called as Reactional Internal Contact Hypothesis hereafter, and it will be described in detail for the case of robotic handwriting in Section II.

There are two main complications to apply this hypothesis 


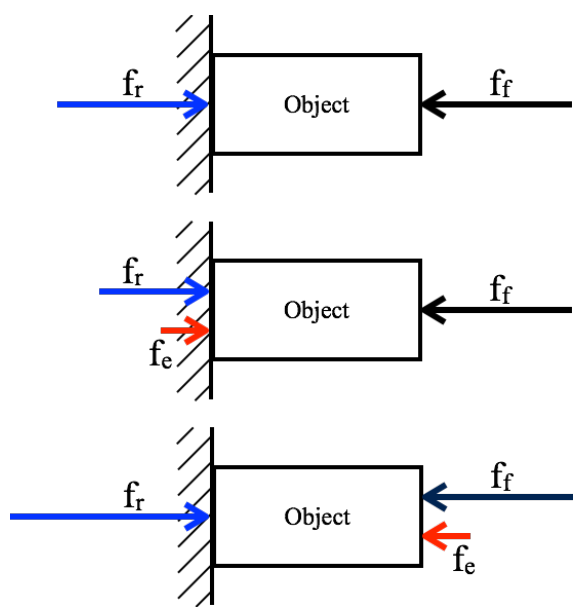

Fig. 2. Visualization of the concept of Reactional Internal Contact Hypothesis. The reaction force from the internal link contact provides resistance to external forces. $f_{f}, f_{r}$, and $f_{e}$ stand for finger force, reaction force, and external force, respectively.

to multi-contact manipulation. One is how to find adequate configurations that satisfies desired contact conditions. The other is, then, how to compute the fingertip force commands for a given task. Section III provides how to find the initial configuration based on workspace and grasp quality metric analysis, and Section IV explains how to compute the grasping forces for the given object end-effector trajectory with some simulation results. Section V concludes this paper with some discussion issues.

\section{REACTIONAL INTERNAL CONTACT HyPOTHESIS}

The implementation framework for Reactional Internal Contact Hypothesis can be applied to general multi-contact manipulation tasks, but in order to develop a concrete problem description, we will take robotic handwriting as the main application.

\section{A. Problem Description}

The problem can be defined as follows:

- Given: A desired path to draw on a paper, predefined grasping taxonomy, i.e., a general handwriting configuration, and initial object information about its geometry and pose.

- Goal: Find a desirable multi-contact grasp configuration for a robotic hand, and draw the given path as a function of time, $t$, on a paper.

- Assumption: The contact condition between the hand and the object is frictional point contact, and the contact point between the object and the internal link, $p_{r}$, does not move with respect to the global frame. The latter assumption is valid if the contacting internal link is the robot base frame, such as the palm, and the wrist remains still during the writing.

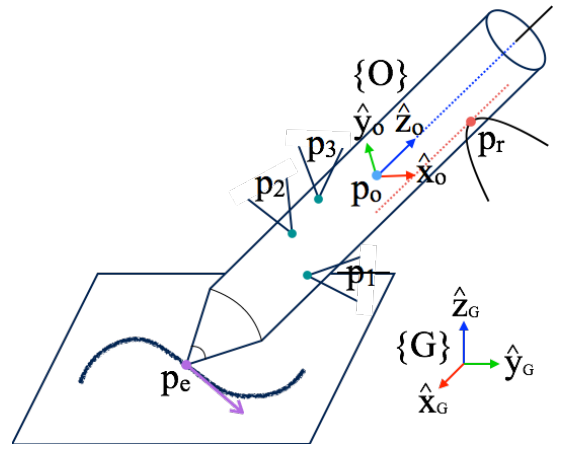

Fig. 3. A schematic diagram for robotic handwriting. $p_{1}, p_{2}, p_{3}$ are fingertip contact points, and $p_{r}$ is a contact point with an internal link. $\hat{z}_{o}$ is along the main axis of the object, $\hat{x}_{o}$ is parallel to the shortest line from $\hat{z}_{o}$ to $p_{r}$.

\section{B. Formulation}

The equation of motion of the object is obtained as

$$
w_{o b j}=\left[\begin{array}{c}
M a_{o b j} \\
I \alpha_{o b j}
\end{array}\right]=G_{e} f_{e}+G_{f} f_{f}+G_{r} \tilde{f}_{r},
$$

or equivallently,

$$
G_{f} f_{f}+G_{r} \tilde{f}_{r}=w_{o b j}-G_{e} f_{e} \triangleq w_{d},
$$

where $G$ is a wrench matrix, $f$ is a linear force applied to the object, and subscript $e, f$, and $r$ imply that they are from environment, fingertip, and reactional internal link, respectively. $w_{o b j}$ is an object wrench composed of 3dimensional force and 3-dimensional moment with respect to the global frame, and $f_{f}=\left[\begin{array}{lll}f_{1}^{T} & f_{2}^{T} & f_{3}^{T}\end{array}\right]^{T}$ is a augmented $9 \times 1$ vector.

The wrench matrix depends on the relative position from the object frame origin to the corresponding contact point as

$$
G_{i}=\left[\begin{array}{c}
I_{3} \\
{\left[p_{o i}\right]_{\times}}
\end{array}\right],
$$

where $p_{o i}=p_{i}-p_{o}$, and $[\cdot]_{\times}$stands for a skewsymmetric matrix for a 3-dimensional vector. Again, $G_{f}=$ $\left[G_{f, 1} G_{f, 2} G_{f, 3}\right]$ is a augmented $6 \times 9$ matrix.

If the contact points and the coordinate systems are assigned as shown in Fig. 3 for clear representation, we can describe the reaction wrench from the internal link contact as follows.

$$
G_{r} \tilde{f}_{r}=\left[\begin{array}{c}
I \\
{\left[p_{o r}\right]_{\times}}
\end{array}\right] \tilde{f}_{r}=\left[\begin{array}{c}
-\xi \hat{x}_{o} \\
-\xi\left\|p_{o r}\right\| \hat{y}_{o}
\end{array}\right],
$$

where $\xi=\left\|\tilde{f}_{r}\right\| \geq 0$.

Note that the magnitude of the reaction force, $\xi$, is unlimited unless the link mechanically breaks down, and the corresponding reaction wrench, $G_{r} \hat{f}_{r}$, is in a fixed direction with respect to the object frame.

\section{Optimization Policy}

In order to find the finger force that satisfies (2) for a given desired wrench, $w_{d}$, it is preferable to find one that uses less energy, in other words, with smaller magnitude. Recall that 
the reaction force can resist the external wrench and help to reduce the required finger force.

Since the finger force has higher number of variables than the number of constraints in the equation of motion, there exist a lot of solutions, and it needs to be optimized [17], [18], [19]. The optimization process should take the frictional contact constraints into account, which is introduced in Section IV and Appendix, and here, we first determine the reaction force that can minimize the magnitude of the finger force.

In general handwriting configuration (and in many multicontact manipulation cases), the fingertip contact points are very close to each other, while the internal contact point and the object end-effector position are relatively far away from them. In that case, the finger force needs to be considerably large to produce a certain mount of moment about the center of the fingertip contact points.

Therefore, the optimization policy for reaction force in this paper is to eliminate the moment for finger force about the fingertip centroid, as long as possible:

$$
\left[\begin{array}{c}
0 \\
\hat{y}_{o}
\end{array}\right]^{T} G_{f}^{\left(p_{c}\right)} f_{f}=0
$$

where $G_{f}^{\left(p_{c}\right)}$ is a wrench matrix about $p_{c}$, the centroid of the fingertip contact points. Note that the reaction force can provide only one directional moment which is in negative- $\hat{y}_{o}$ direction under the object frame convention as in Fig. 3.

Equation (2) and (5) yield that the magnitude of the reaction force satisfies the following to minimize the finger force for a desired wrench.

$$
\xi^{*}=\max \left(0, \frac{\left\|p_{e}-p_{c}\right\|}{\left\|p_{r}-p_{c}\right\|} \hat{x}_{o}^{T} U w_{d}\right) \geq 0
$$

where $p_{c}=\frac{1}{n_{f}} \sum_{i=1}^{n_{f}} p_{i}$ for $n_{f}=3$, and $U=\left[\begin{array}{ll}I_{3} & O_{3}\end{array}\right]$.

As a result, a reduced desired wrench is obtained by

$$
\begin{aligned}
\tilde{w}_{d} & \triangleq w_{d}-G_{r} \tilde{f}_{r}=G_{f} f_{f} \\
& = \begin{cases}w_{d} & \left(\hat{x}_{o}^{T} U w_{d} \leq 0\right) \\
\left(I+G_{r} \frac{\left\|p_{e}-p_{c}\right\|}{\left\|p_{r}-p_{c}\right\|} \hat{x}_{o} \hat{x}_{o}^{T} U\right) w_{d} & \left(\hat{x}_{o}^{T} U w_{d}>0\right)\end{cases}
\end{aligned}
$$

This equation shows that the reactional internal contact comes into effect when the desired wrench is in the opposite direction to the reaction force. It is because, in the handwriting configuration, the object end-effector and the internal contact point lie on the opposite side with respect to the fingertip contact points.

\section{Initial CONFIGURATION SEARCH}

This section describes how to find the suitable initial configuration for robotic handwriting. There are two main processes. The first is common workspace search process to register the robot base to a given object, and the second is optimal configuration search process to register the individual fingertips on the object surface.

\section{A. Common Workspace Search}

For the given object geometry and position/orientation, the robot hand needs to be located properly with respect to the object. The basic idea here is to register the robot so that the object lies in the center of common workspace of the fingers.

1) Determine the Internal Contact Position: Since it is assumed that the internal link contact position is fixed for the given multi-contact taxonomy, we can determine the internal contact point manually for handwriting taxonomy that associates three fingertip contacts and one internal contact.

For a robotic hand in Fig. 4(a) as an example, the internal contact point is decided as the base joint of the index finger. Note that, for the graphical convenience, we will represent the figures in the robot hand frame instead of the global frame, which makes the object seem to be registered to the robot.

2) Generate Uniform Workspace Samples: By taking steps in the joint space, uniform workspace samples are generated for individual fingers. Table I shows the joint limit of the thumb and fingers, and Fig. 4(b) presents the end-effector position of each sample.

3) Find the Center of Common Workspace: The center of the common workspace can be found by a numerical method [21]. First, find the common workspace by tessellating the Cartesian space into a set of small cells and checking each cell for existence of all samples of the thumb and fingers, and then, compute the centroid of the common workspace cells.

4) Register the Hand to the Object: Now we have two points; internal link contact point and center of the common workspace. Place the robot hand so that they lie on the main axis of the pen-type (cylindrical) object. Fig. 4(c) shows the result of the robot hand registration to the given object.

\section{B. Optimal Configuration Search}

Now we need to find the fingertip contact points on the object surface that offer the best grasp quality for the object.

TABLE I

JOINT LIMIT OF THUMB AND FINGERS

\begin{tabular}{c|c|c}
\hline \hline & Joint & Limit [deg] \\
\hline \multirow{4}{*}{ Thumb } & 1 & {$[-110,10]$} \\
& 2 & {$[-45,45]$} \\
& 3 & {$[-10,80]$} \\
& 4 & {$[-10,80]$} \\
\hline \multirow{4}{*}{ Fingers } & 1 & {$[-30,30]$} \\
& 2 & {$[-10,80]$} \\
& 3 & {$[-10,80]$} \\
& 4 & {$[-10,80]$} \\
\hline \hline
\end{tabular}




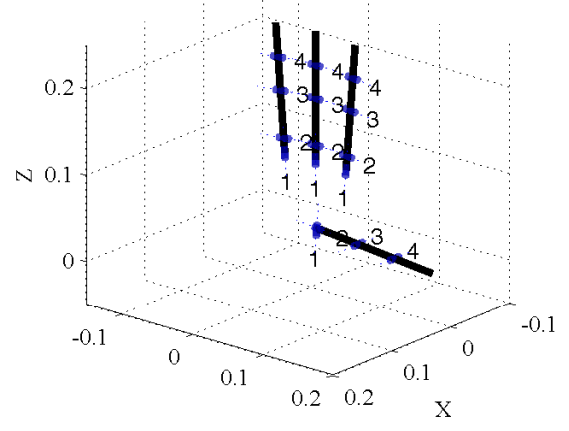

(a) Robot hand in home configuration

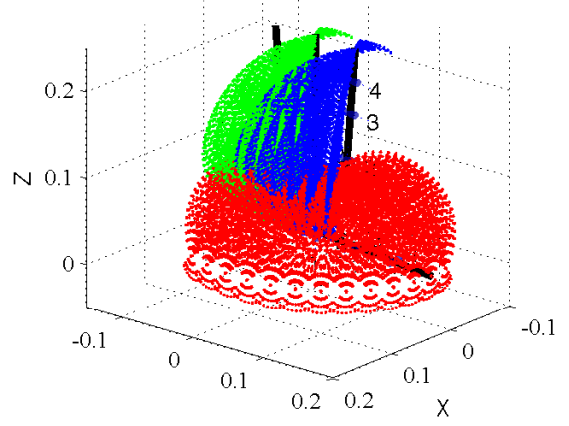

(b) Uniform workspace samples

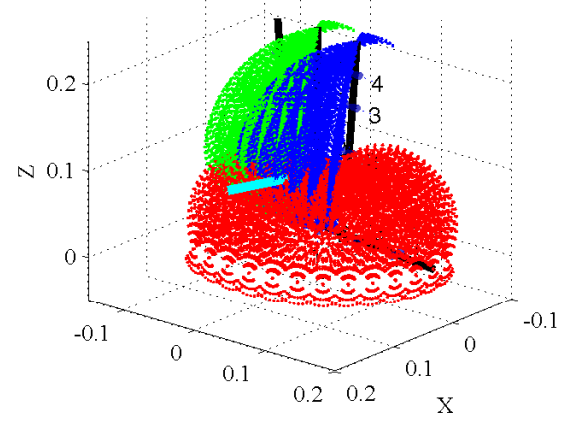

(c) Object at the center of common workspace

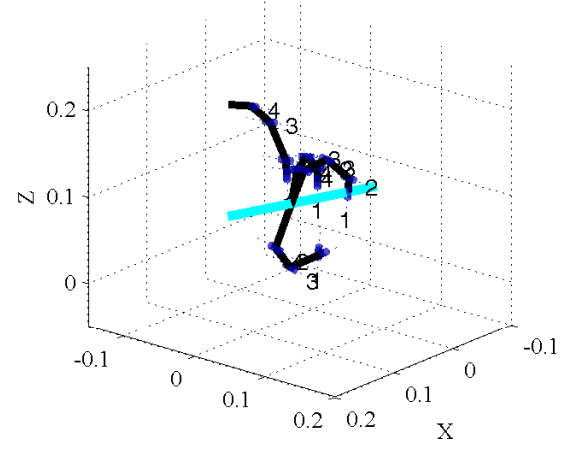

(d) Nominal finger configuration for the object

Fig. 4. Common workspace search process for robot hand registration to the object. The figures are depicted using MATLAB Robotics Toolbox [20].
Before introducing the optimal configuration search process, let us define the grasp quality metric, $Q_{g}$, in the multi-contact manipulation setting.

Bicchi has pointed out that the manipulability index is the ratio of the output performance to the input effort, in both velocity and force aspects [22], [23]. At that point of view, the force manipulability can be defined as

$$
R=\frac{w_{d}^{T} W_{w} w_{d}}{\sum_{i} f_{f, i}^{T} W_{f} f_{f, i}},
$$

where $W_{w}$ and $W_{f}$ are positive definite weighting matrices for desired object wrench and finger force, respectively.

As an extension to the multi-contact manipulation with redundancy, passive manipulability with optimization is defined as follows.

$$
\begin{aligned}
R_{o p t}^{p}\left(\hat{w}_{d}\right) & =\max _{f_{f}} \frac{\hat{w}_{d}^{T} W_{w} \hat{w}_{d}}{\sum_{i} f_{f, i}^{T} W_{f} f_{f, i}}, \\
f_{f}^{*}\left(\hat{w}_{d}\right) & =\underset{f_{f}}{\operatorname{argmin}} \sum_{i} f_{f, i}^{T} W_{f} f_{f, i} .
\end{aligned}
$$

For a desired output wrench, $\hat{w}_{d}$, the optimization process finds $f_{f}^{*}$ that minimizes the sum of finger force magnitudes and yields the maximum value of the ratio as $R_{o p t}^{p}$. Note that the optimization process incorporates Reactional Internal Contact Hypothesis as well as the friction constraints.

$\hat{w}_{d} \in \mathbb{W}_{d}$ is a basis vector of the object wrench space which is generated by a unit force applied at the end-effector.

$$
\begin{aligned}
\mathbb{W}_{d} & =\left(\hat{w}_{d}\right)_{j}=\left\{\hat{w}_{d} \mid \hat{w}_{d}=G_{e} \hat{f}_{d}, \hat{f}_{d} \in \mathbb{F}_{d}\right\}, \\
\mathbb{F}_{d} & =\left\{ \pm \hat{e}_{x}, \pm \hat{e}_{y}, \pm \hat{e}_{z}\right\},
\end{aligned}
$$

where $\hat{e}_{k}$ is a unit vector along the $k$-axis in the global frame. Notice that the number of basis vectors is six in threedimensional space because the finger force to the object is unilateral, not bilateral.

The grasp quality metric, $Q_{g}$, for a given fingertip contact configuration, $p_{f}=\left[\begin{array}{lll}p_{1}^{T} & p_{2}^{T} & p_{3}^{T}\end{array}\right]^{T}$, can be defined using the passive force manipulability as

$$
Q_{g}\left(p_{f}\right)=\frac{1}{n_{d}} \sum_{j=1}^{n_{d}} R_{o p t}^{p}\left(\hat{w}_{d, j}\right),
$$

where $n_{d}=6$.

The followings are the sequential steps in the optimal configuration search process.

1) Generate Uniform Configuration Samples: The first step is to generate uniform configuration samples, i.e., fingertip contact point samples, to examine the grasp quality metric. Centered at the nominal configuration which lies in the midst of the common workspace as in Fig. 4(d), the contact point samples on the object surface are generated. In this example, we sampled 8 sets in radial coordinate and 5 sets in axial coordinate, which results in 64,000 samples in total for three fingers.

2) Select Samples without Collision: Since the computation of grasp quality metric is expensive, we need to eliminate the meaningless samples beforehand. The 
samples that incur collision between finger-and-finger or finger-and-object are filtered out by geometric criteria. This step reduces the number of samples to 2,500 in the example.

3) Compute Grasp Quality Metric: For each configuration samples, the grasp quality metric is computed in this step. Figure 5(a) shows the average magnitude of $f_{f}^{*}$ over $\hat{w}_{d, j}$ for each configuration sample, and Fig. 5(b) presents how many basis wrenches can be achieved in that configuration. Excluding the degenerate samples, the computed $Q_{g}$ is depicted in Fig. 5(c).

4) Determine the Optimal Configuration: Finally, we select the configuration that has the maximum $Q_{g}$ as our initial configuration for multi-contact manipulation. The result is shown in Fig. 5(d). It would seem to be a little different from human's configuration because of the kinematic discrepancy between them.

This searching process can be applied to other multicontact manipulation tasks by selecting the corresponding grasp taxonomy and the internal link contact point.

\section{GRASPING Force COMPUTATION}

A grasping force optimization algorithm for handwriting is presented in this section. The basic algorithms can be found in [17], [24], [25], while the main contribution of this paper is to adopt them to the multi-contact manipulation tasks under Reactional Internal Contact Hypothesis.

The following steps are executed for every control loop.

1) Compute a Desired Wrench: For a given writing trajectory for the object end-effector, $w_{o b j}$ and $f_{e}$ are computed as follows to obtain $w_{d}$.

$$
w_{o b j}=\left[\begin{array}{c}
M a_{o b j} \\
I \alpha_{o b j}
\end{array}\right]=\left[\begin{array}{c}
M \frac{\left\|p_{o}-p_{r}\right\|}{\left\|p_{e}-p_{r}\right\|} \frac{d^{2}}{d t^{2}} p_{e}(t) \\
I \frac{d}{d t} \omega_{o b j}(t)
\end{array}\right],
$$

where $p_{e}(t)$ is the given end-effector trajectory, and $\omega_{o b j}$ is the object angular velocity obtained from kinematic constraints for $p_{e}$ and $p_{r}$.

Based on the Coulomb-viscous friction model,

$$
\begin{aligned}
f_{e} & =f_{e, n}+f_{e, t} \\
& =\bar{f}_{e, n} \hat{e}_{z}-\left(c_{0}\left\|\frac{d}{d t} p_{e}(t)\right\|^{-1}+c_{v}\right) \frac{d}{d t} p_{e}(t),
\end{aligned}
$$

where $\bar{f}_{e, n}$ is a constant that satisfies $0 \leq \bar{f}_{e, n}<\frac{c_{0}}{\mu_{o b j}}$, and $c_{0}$ and $c_{v}$ are friction coefficients between the object and the paper. Note that $f_{e}$ is defined as a force applied to the object. Now, we can compute $w_{d}$ that the robotic hand needs to exert to the object using (2).

2) Compute the Reduced Desired Wrench: As discussed in Section II, Reactional Internal Contact Hypothesis suggests that the internal contact force can help to reduce the desired wrench for the fingers. Equation (8) gives the reduced desired wrench, $\tilde{w}_{d}$, from the original desired wrench, $w_{d}$.

3) Find the Optimal Finger Force: This paper employs linear approximation for the friction constraints and

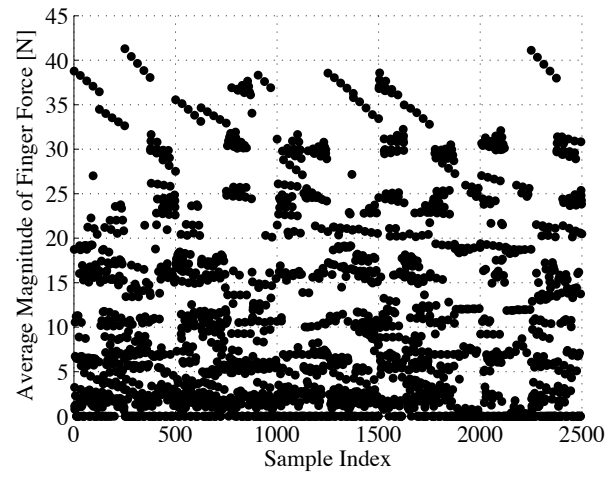

(a) Average magnitude of finger force

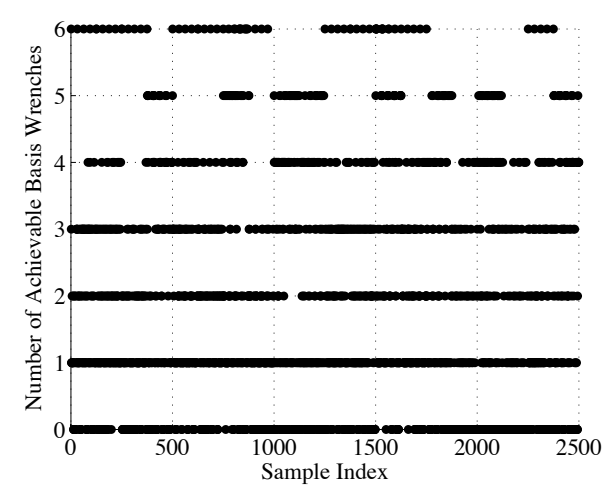

(b) Number of achievable basis wrenches

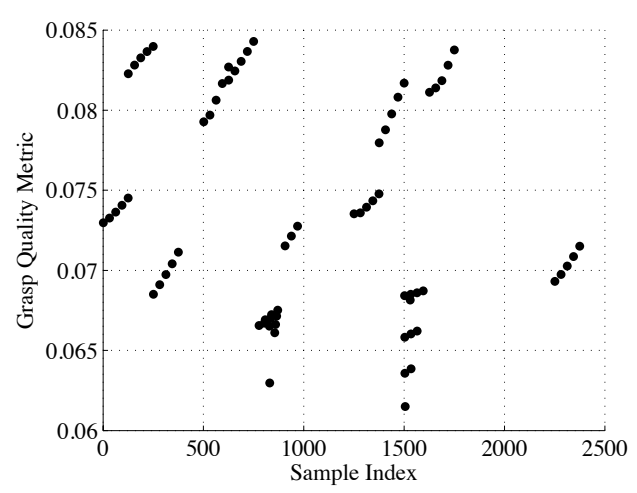

(c) Grasp quality metric for non-degenerate samples

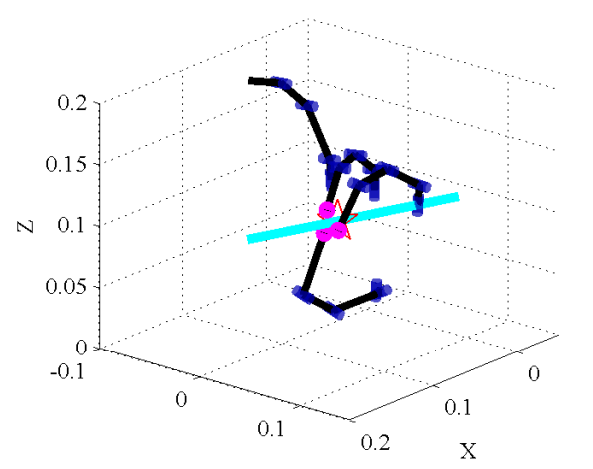

(d) Optimal finger configuration for the object

Fig. 5. Optimal configuration search process for fingertip registration on the object surface. 
develops the finger force optimization as a linear programming problem. For readers who are interested in advanced methods for grasping force optimization problem, see [18], [19].

As provided in Appendix, the friction cone and the maximum finger force constraints can be represented in a matrix inequality form as

$$
A B f_{f} \geq P
$$

and the general solution for (2) is

$$
f_{f}=G_{f}^{\#} \tilde{w}_{d}+N_{f} \lambda,
$$

where $N_{f}$ consists of orthonormal basis vectors of the null space of $G_{f}$, and $\lambda$ is a $3 \times 1$ variable vector in our example unless $G_{f}$ is non-degenerate.

Then, (18) can be rewritten as

$$
A B N_{f} \lambda \geq P-A B G_{f}^{\#} \tilde{w}_{d},
$$

and we want to find $\lambda$ that provides the largest margins to the constraints. The margin for each constraint can be represented by one non-negative parameter, $d$, with some weighting factor. By augmenting $\lambda$ with $d$, a new constraint inequality with an optimization parameter can be obtained.

$$
\begin{aligned}
& {\left[\begin{array}{c|c}
A B N_{f} & -W_{d} \\
\hline 0 & 1
\end{array}\right] } {\left[\frac{\lambda}{d}\right] \geq\left[\frac{P-A B G_{f}^{\#} \tilde{w}_{d}}{0}\right], } \\
&\left(\tilde{A} \lambda_{d} \geq \tilde{P}\right)
\end{aligned}
$$

where $W_{d}=\left[\begin{array}{lll}w_{1}^{T} & w_{2}^{T} & w_{3}^{T}\end{array}\right]^{T}$ for $w_{i}=\left[\begin{array}{llllll}0 & \frac{1}{\mu} & 1 & 1 & 1 & 1\end{array}\right]^{T}$.

Now, we can form a linear programming problem as

$$
\begin{aligned}
& \operatorname{maximize} F\left(\lambda_{d}\right) \\
& \text { subject to } \tilde{A} \lambda_{d} \geq \tilde{P},
\end{aligned}
$$

where $F\left(\lambda_{d}\right)=\left[\begin{array}{llll}0 & 0 & 0 & 1\end{array}\right]\left[\begin{array}{l}\lambda \\ d\end{array}\right]=d$. As well known, linear programming problems are readily solved. From the solution, $\lambda_{d}^{*}$, for (22), the optimal finger force can be obtained, and the joint torque for each finger robot is computed as in [26].

For validation of the proposed method, dynamic simulation was conducted. As shown in Fig. 6, two experiments are presented in this paper: drawing a simple triangle and the logo of the Robotics Institute. The detail results are illustrated in Fig. 7 and Fig. 8, respectively. The one thing that should be noted is that the magnitude of finger force gets much smaller when Reactional Internal Contact Hypothesis takes effect.

\section{CONCLUSiON}

In this paper, a novel framework for multi-contact manipulation is proposed based on Reactional Internal Contact Hypothesis that views the internal link contact force as a reaction force from an immobile environment. In this framework, we can utilize the internal link force as a part of

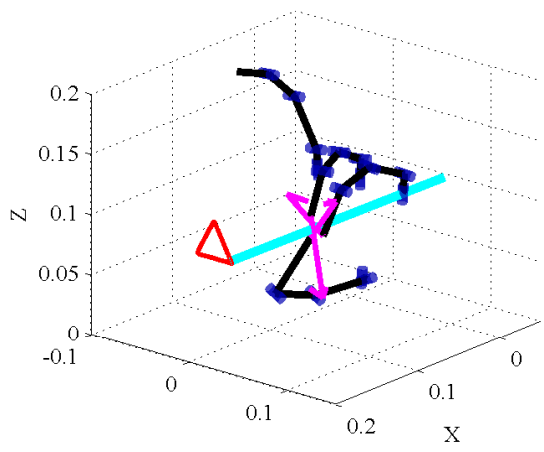

(a) Drawing a simple triangle

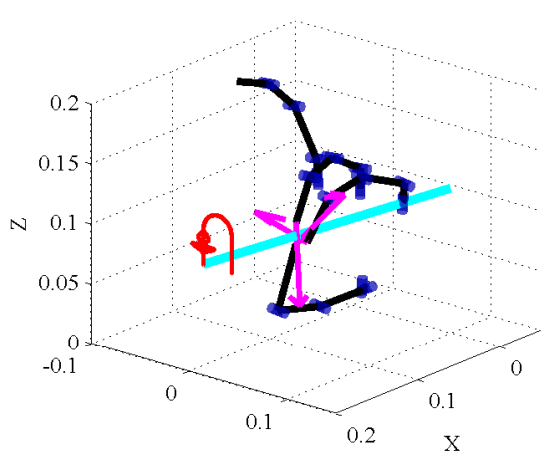

(b) Drawing the logo of the Robotics Institute

Fig. 6. Dynamic simulation of robotic handwriting (see the attached video)

strong grasping wrench, without knowledge about the value of the force.

The two main problems to apply this idea to multi-contact manipulation are addressed: finding the optimal initial configuration using grasp quality metric, and computing the optimal grasping force. The key idea of Reactional Internal Contact Hypothesis is formulated by the derivation of reduced desired wrench is and validated by dynamic simulation of handwriting tasks.

As a future work, it is needed to accommodate the robot dynamics into the computation process of grasping force. This paper only deals with the motion of the object by assuming that the robot dynamics is considerably fast compared to that of the object, but it may not be the case in reality. Thus, it would be required to consider the closed-loop dynamics of the finger robots and the object.

\section{REFERENCES}

[1] Mark Cutkosky, "On grasp choice, grasp models, and the design of hands for manufacturing tasks", IEEE Transactions on Robotics and Automation, vol. 5, no. 3, pp. 269-279, 1989.

[2] Matthew T Mason, Alberto Rodriguez, Siddhartha S Srinivasa, and Andrés S Vazquez, "Autonomous manipulation with a general-purpose simple hand", The International Journal of Robotics Research, vol. 31, no. 5, pp. 688-703, 2012.

[3] Andrew T Miller, Steffen Knoop, Henrik I Christensen, and Peter K Allen, "Automatic grasp planning using shape primitives", in Proceedings of IEEE International Conference on Robotics and Automation (ICRA), 2003, vol. 2, pp. 1824-1829. 


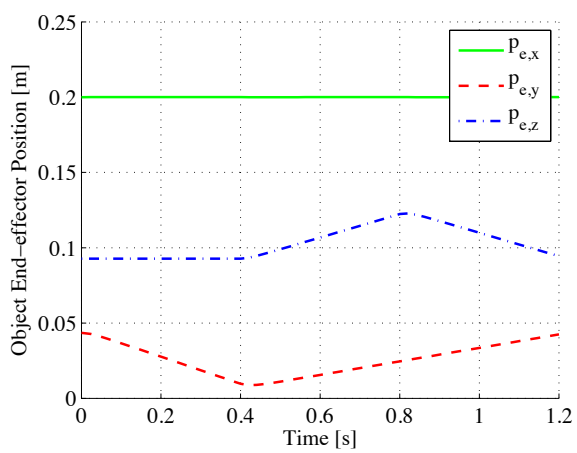

(a) Object end-effector position.

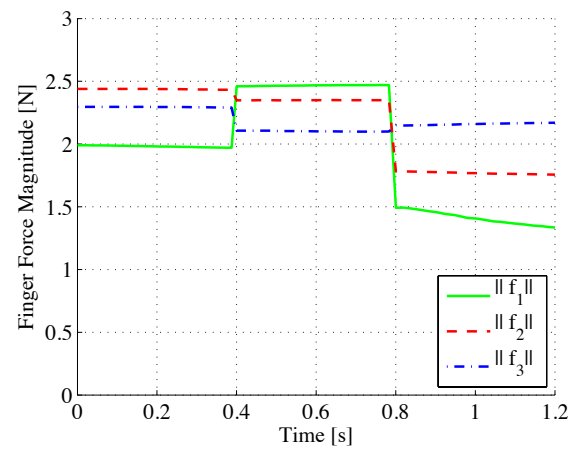

(b) Finger force magnitude (without RICH)

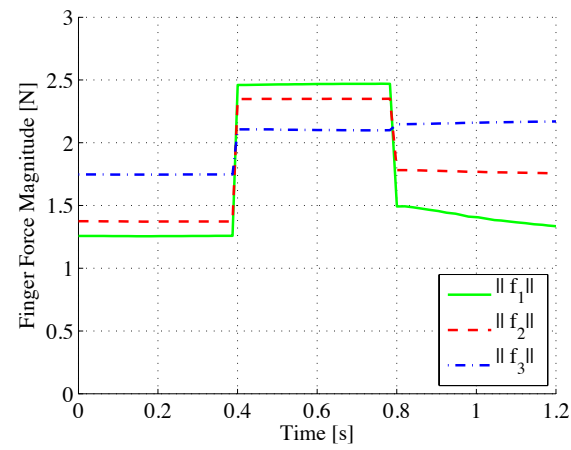

(c) Finger force magnitude (with RICH)

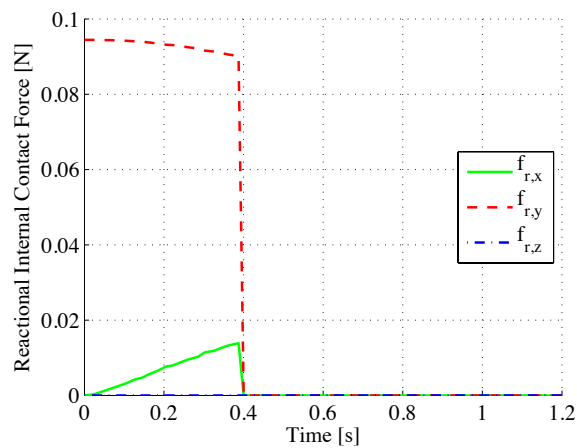

(d) Reactional internal contact force (with RICH)

Fig. 7. Drawing a simple triangle on a plane at $x=0.2$. RICH is the abbreviation of Reactional Internal Contact Hypothesis. Note that RICH is only operative when the end-effector moves in the opposite direction that the internal force can be exerted but gives significant effect in reducing the required finger force for the same motion.

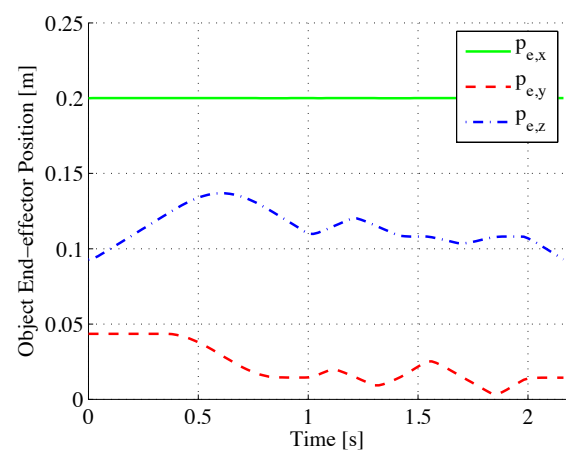

(a) Object end-effector position.

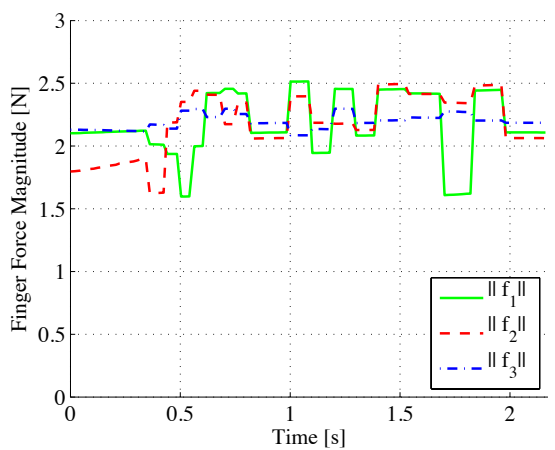

(b) Finger force magnitude (without RICH)

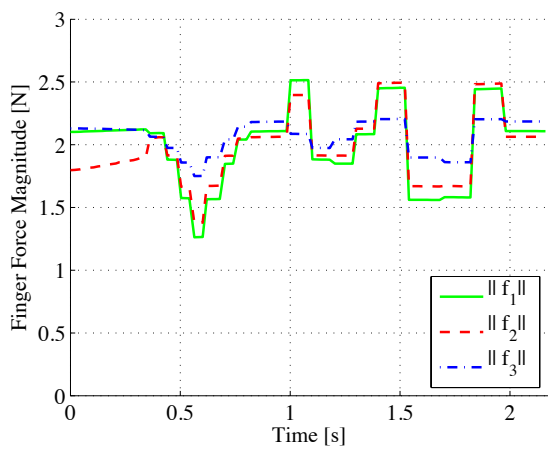

(c) Finger force magnitude (with RICH)

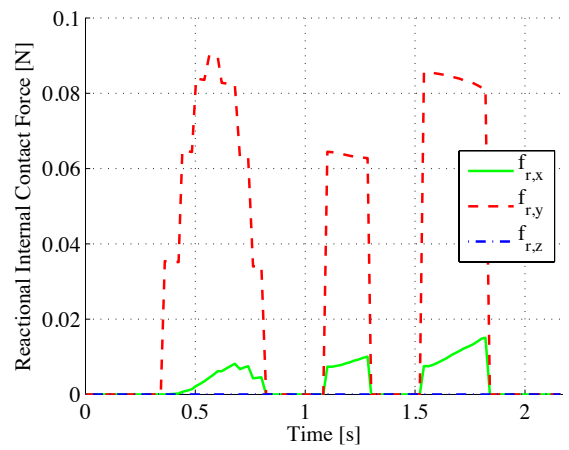

(d) Reactional internal contact force (with RICH)

Fig. 8. Drawing the $\log$ of the Robotics Institute on a plane at $x=0.2$. This task is composed of more complicated sequence of motions, but Reactional Internal Contact Hypothesis plays the role to reduce the finger force magnitude when it is operative. 
[4] Kaijen Hsiao, Sachin Chitta, Matei Ciocarlie, and Edward Gil Jones, "Contact-reactive grasping of objects with partial shape information", in Proceedings of IEEE/RSJ International Conference on Intelligent Robots and Systems (IROS), 2010, pp. 1228-1235.

[5] Aaron M Dollar and Robert D Howe, "Simple, robust autonomous grasping in unstructured environments", in Proceedings of IEEE International Conference on Robotics and Automation (ICRA), 2007, pp. 4693-4700.

[6] Dennis Hong, Colin Smith, Alex McCraw, Carlos Guevara, and Cothern Kyle, "Design of a robotic air-powered hand with elastic ligaments", in Proceedings of International Conference on Ubiquitous Robots and Ambient Intelligence (URAI), 2009.

[7] Jonna Laaksonen, Ekaterina Nikandrova, and Ville Kyrki, "Probabilistic sensor-based grasping", in Proceedings of IEEE/RSJ International Conference on Intelligent Robots and Systems (IROS), 2012, pp. 20192026.

[8] Anna Kochan, "Shadow delivers first hand", Industrial robot: an international journal, vol. 32, no. 1, pp. 15-16, 2005.

[9] CS Lovchik and Myron A Diftler, "The robonaut hand: A dexterous robot hand for space", in Proceedings of IEEE International Conference on Robotics and Automation (ICRA), 1999, vol. 2, pp. 907-912.

[10] Jörg Butterfaß, Markus Grebenstein, Hong Liu, and Gerd Hirzinger, "Dlr-hand ii: Next generation of a dextrous robot hand", in Proceedings of IEEE International Conference on Robotics and Automation (ICRA), 2001, vol. 1, pp. 109-114.

[11] Thomas Schlegl, Martin Buss, Toru Omata, and Günther Schmidt, "Fast dextrous re-grasping with optimal contact forces and contact sensor-based impedance control", in Proceedings of IEEE International Conference on Robotics and Automation (ICRA). IEEE, 2001, vol. 1, pp. 103-108.

[12] Masahiro Ohka, Hiroaki Kobayashi, and Yasunaga Mitsuya, "Sensing characteristics of an optical three-axis tactile sensor mounted on a multi-fingered robotic hand", in Proceedings of IEEE/RSJ International Conference on Intelligent Robots and Systems (IROS). IEEE, 2005, pp. 493-498.

[13] Mark Moll and Michael A Erdmann, "Reconstructing the shape and motion of unknown objects with active tactile sensors", in Algorithmic Foundations of Robotics V, pp. 293-310. Springer, 2004.

[14] Giorgio Cannata, Marco Maggiali, Giorgio Metta, and Giulio Sandini, "An embedded artificial skin for humanoid robots", in Proceedings of IEEE International Conference on Multisensor Fusion and Integration for Intelligent Systems. IEEE, 2008, pp. 434-438.

[15] Anna Petrovskaya, Jaeheung Park, and Oussama Khatib, "Probabilistic estimation of whole body contacts for multi-contact robot control", in Proceedings of IEEE International Conference on Robotics and Automation (ICRA), 2007, pp. 568-573.

[16] Andrea Bajo and Nabil Simaan, "Finding lost wrenches: Using continuum robots for contact detection and estimation of contact location", in Proceedings of IEEE International Conference on Robotics and Automation (ICRA). IEEE, 2010, pp. 3666-3673.

[17] Jeffrey Kerr and Bernard Roth, "Analysis of multifingered hands", The International Journal of Robotics Research, vol. 4, no. 4, pp. 3-17, 1986.

[18] Martin Buss, Hideki Hashimoto, and John B Moore, "Dextrous hand grasping force optimization", IEEE Transactions on Robotics and Automation, vol. 12, no. 3, pp. 406-418, 1996.

[19] Vincenzo Lippiello, Bruno Siciliano, and Luigi Villani, "A grasping force optimization algorithm for multiarm robots with multifingered hands", IEEE Transactions on Robotics, vol. 29, no. 1, pp. 55-67, 2013.

[20] Peter I. Corke, Robotics, Vision \& Control: Fundamental Algorithms in Matlab, Springer, 2011.

[21] D Alciatore and $\mathrm{C} \mathrm{Ng}$, "Determining manipulator workspace boundaries using the monte carlo method and least squares segmentation", ASME Robotics: Kinematics, Dynamics and Control, vol. 72, pp. 141146, 1994.

[22] Antonio Bicchi, Claudio Melchiorri, and Daniele Balluchi, "On the mobility and manipulability of general multiple limb robots", IEEE Transactions on Robotics and Automation, vol. 11, no. 2, pp. 215-228, 1995.

[23] Antonio Bicchi and Domenico Prattichizzo, "Manipulability of cooperating robots with unactuated joints and closed-chain mechanisms", IEEE Transactions on Robotics and Automation, vol. 16, no. 4, pp. 336-345, 2000.
[24] Robert Bonitz and Tien Hsia, "Force decomposition in cooperating manipulators using the theory of metric spaces and generalized inverses", in Proceedings of IEEE International Conference on Robotics and Automation (ICRA), 1994, pp. 1521-1527.

[25] David Williams and Oussama Khatib, "The virtual linkage: A model for internal forces in multi-grasp manipulation", in Proceedings of IEEE International Conference on Robotics and Automation (ICRA), 1993, pp. 1025-1030.

[26] Sung-Kyun Kim, Yonghwan Oh, and Sang-Rok Oh, “Object manipulation in $3 \mathrm{~d}$ space by two cone-shaped finger robots based on fingerthumb opposability without object sensing", in Proceedings of IEEE International Conference on Robotics and Automation (ICRA). IEEE, 2012, pp. 5136-5141.

\section{APPENDIX}

This section presents how to form a matrix inequality from the friction and maximum force constraints.

Recall that, in the frictional point contact condition, a force should remain inside the friction cone to keep in contact without slippage. For $f_{f, i}=-c_{r, i} \hat{e}_{r}+c_{\theta, i} \hat{e}_{\theta}+c_{z, i} \hat{e}_{z}$ which is represented in the cylindrical coordinate of the object, friction constraint inequalities are as follows.

$$
\begin{aligned}
c_{r, i} & \geq 0, \\
\sqrt{c_{\theta, i}^{2}+c_{z, i}^{2}} & \leq \mu c_{r, i},
\end{aligned}
$$

where $\mu$ is a friction coefficient between the finger and the object. By applying linear approximation to (A.2), we have

$$
\begin{aligned}
-\mu c_{r, i} & \leq c_{\theta, i} \leq \mu c_{r, i}, \\
-\mu c_{r, i} & \leq c_{z, i} \leq \mu c_{r, i} .
\end{aligned}
$$

In addition, we can impose maximum finger force constraint.

$$
c_{r, i} \leq c_{r, \max }
$$

where $c_{r, \max }$ is a constant.

Then, these inequalities can be rewritten as a matrix form,

$$
A_{i} C_{i} \geq P_{i}
$$

where $C_{i}=\left[c_{r, i}^{T} c_{\theta, i}^{T} c_{z, i}^{T}\right]^{T}$. Using the following equation,

$$
\begin{gathered}
{\left[\begin{array}{c}
c_{r, i} \\
c_{\theta, i} \\
c_{z, i}
\end{array}\right]=\left[\begin{array}{c}
-\hat{e}_{r}^{T} \\
\hat{e}_{\theta}^{T} \\
\hat{e}_{z}^{T}
\end{array}\right] f_{f, i},} \\
\left(C_{i}=B_{i} f_{f, i}\right)
\end{gathered}
$$

we can construct a matrix inequality for $f_{f, i}$ as

$$
\begin{array}{r}
{\left[\begin{array}{ccc}
1 & 0 & 0 \\
-1 & 0 & 0 \\
\mu & 1 & 0 \\
\mu & -1 & 0 \\
\mu & 0 & 1 \\
\mu & 0 & -1
\end{array}\right]} \\
\left(A_{i} B_{i} f_{f, i} \geq P_{i}\right)
\end{array}
$$

For $f_{f}=\left[\begin{array}{lll}f_{f, 1}^{T} & f_{f, 2}^{T} & f_{f, 3}^{T}\end{array}\right]^{T}$, we can build block diagonal matrices, $A$ and $B$, and a concatenated vector, $P$, from (A.8), which gives rise to

$$
A B f_{f} \geq P .
$$

